\title{
Towards a normalised 3D geovisualisation: The viewpoint management
}

\author{
R. Neuville ${ }^{\text {a }}$ F. Poux ${ }^{\text {a }}$, P.Hallot ${ }^{\text {a }}$, R. Billen ${ }^{\text {a }}$ \\ ${ }^{a}$ Geomatics Unit, Department of Geography, University of Liège, Allée du Six Aout, Belgium - (romain.neuville, fpoux, p.hallot, \\ rbillen)@ulg.ac.be
}

KEY WORDS: 3D geovisualisation, 3D cartography, 3D semiotics, camera management, visual variables, user's query

\begin{abstract}
:
This paper deals with the viewpoint management in 3D environments considering an allocentric environment. The recent advances in computer sciences and the growing number of affordable remote sensors lead to impressive improvements in the 3D visualisation. Despite some research relating to the analysis of visual variables used in 3D environments, we notice that it lacks a real standardisation of 3D representation rules. In this paper we study the "viewpoint" as being the first considered parameter for a normalised visualisation of 3D data. Unlike in a 2D environment, the viewing direction is not only fixed in a top down direction in 3D. A non-optimal camera location means a poor 3D representation in terms of relayed information. Based on this statement we propose a model based on the analysis of the computational display pixels that determines a viewpoint maximising the relayed information according to one kind of query. We developed an OpenGL prototype working on screen pixels that allows to determine the optimal camera location based on a screen pixels colour algorithm. The viewpoint management constitutes a first step towards a normalised 3D geovisualisation.
\end{abstract}

\section{INTRODUCTION}

The advances in computer sciences in terms of storage and processing of large amount of data and the growing number of acquisition techniques (LiDAR, photogrammetry and remote sensing) of the last decade led to impressive improvements in the $3 \mathrm{D}$ visualisation. 3D models are used in various application fields such as environmental modelling, risk management, city planning, urban visualisation, indoor navigation, teaching, analysis, demonstrations ... (Bandrova, 2005; Häberling et al., 2008).

Nevertheless, the way to visualise 3D geospatial data is far from being normalised. Indeed, 3D representation techniques are numerous and depend on the concerned application fields (Métral et al., 2014) which limits their use. This is why a standardisation of representation rules must be developed for 3D geovisualisation. Our research aims at studying and standardising the parameters used to represent and visualise all 3D realities that include geospatial data (see figure 1). This paper addresses the viewpoint management as a first step towards a normalised 3D geovisualisation. The camera management is specific to allocentric views since the relayed information is independent of the user's movement. Therefore, the viewpoint management is essential otherwise the view will be a poor representation of the $3 \mathrm{D}$ environment in terms of relayed information. At the moment, it is worth noting that we only consider the outside viewpoint of objects.

This paper is organised as follows. Section 2 presents the 3D geovisualisation context. Section 3 presents the adopted approach, a case study dealing with the viewpoint and the obtained results. A discussion of the results is provided in section 4. Finally we conclude and address perspectives for future works.

\section{3D GEOVISUALISATION}

\subsection{Context}

According to Häberling and Baer, the term $3 D$ map is used as a computer-generated perspective view of a three-dimensional geo-data model with cartographic components presented on twodimensional media such as computer display or a paper display (Häberling and Baer, 2006) ${ }^{1}$. Unlike in 2D, 3D maps introduce new geometric aspects: e.g. perspective distortions, infinite number of scales on a same scene. These characteristics can be taken as advantages for the development of a naïve geography and for the spatial relations understanding (Jobst and Germanchis, 2007; Jobst and Döllner, 2008). According to Egenhofer and Mark, naïve geography refers to the instinctive reason about geographical and temporal phenomena (Egenhofer and Mark, 1995). Indeed, the infinite number of scales enables to reconstruct a natural human aspect while dynamic maps enable to move (translation and rotation) in a 3D natural environment where movement metaphors (walking, head movement) are then reproduced (Jobst and Döllner, 2008).

The level of 3D perception can be used to establish a 3D maps taxonomy. First, a DTM (Digital Terrain Model) can be draped by a topographic map or an orthophoto. A 3D symbolisation can be added to the DTM although it remains a 3D objects visualisation on a 2D medium. These two kinds of representation are also called 2.5D maps. On the other hand, real "3D maps" use holograms to represent the landscape (Petrovič and Mašera, 2005).

The needs and the development for 3D maps are linked to the limitations of 3D photorealistic representations: they do not easily enable to represent and extract thematic information. Moreover, photorealistic representations cannot introduce different states in the scene (like removed, existing and planned objects) due to the lack of graphics styles (e.g. sketchy and outlined drawings). Finally, a photorealistic representation is not adapted (due to its storage size) to be visualised on devices with low capacities like mobile phones (Jobst et al., 2008). Ellul and 
Altenbuchner show that an aggregation and simplification process is necessary to efficiently visualise 3D data sets on devices with low performance like mobile phones and tablets (Ellul and Altenbuchner, 2014).

3D maps are widely used both by the end-users and professionals in numerous applications (environmental and city modelling, navigation...) which implies the need to develop a standardisation of $3 \mathrm{D}$ representation rules. Nevertheless, the advances in computer sciences and the growing number of acquisition techniques allowed the development of $3 \mathrm{D}$ applications that include geospatial data located in the virtuality continuum (see figure 1) from Real to Virtual Environments and Mixed Reality.

Real and virtual environments are realities (respectively) constituted of objects solely real or virtual. Between real and virtual environments stand the Augmented Reality and Virtuality that belong to the Mixed Reality. Augmented reality is characterized by a real environment supplemented by virtual objects through computer graphics (Milgram and Kishino, 1994). In an augmented reality, real and virtual objects present rational spatial relations and coexist in an augmented space (Behzadan $e t$ al., 2015). An augmented world mainly created by computerbased techniques on a real environment is referred as an augmented virtuality (Milgram and Kishino, 1994).

Augmented virtuality and augmented reality can either be immersive or non-immersive depending on the type of display. The immersion can be obtained with the use of a head-mounted display or a CAVE (Cave Automatic Virtual Environment) since a sheet of paper or a monitor-based display provides a nonimmersive view (Milgram et al., 1995). The augmented world can then be observed via a monoscopic vision (via paper or a screen) or stereoscopic vision (via glasses, head-mounted displays or a CAVE). Finally, the views can either be qualified as egocentric or allocentric (exocentric).

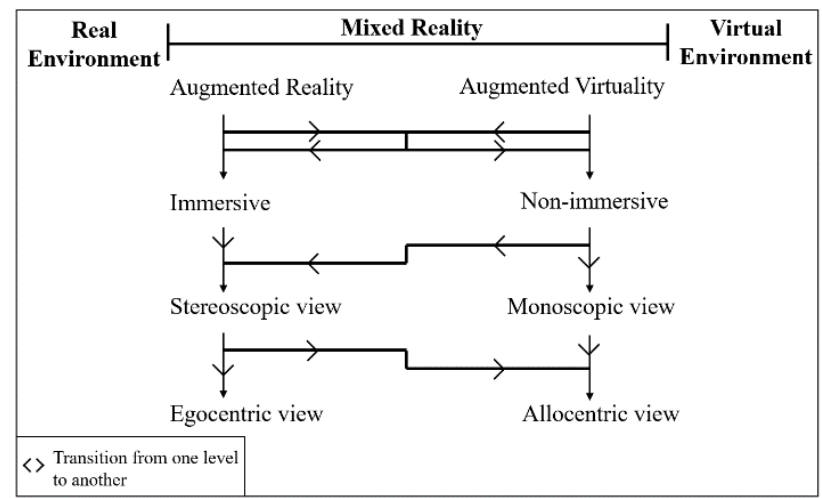

Figure 1. Inspired from Milgram's virtuality continuum.

Arrows represent the transition from one level to another (Milgram, 1994)

In conclusion, the visualisation of 3D geospatial data does not only concern the 3D cartography but also 3D applications (located in the virtual continuum) that include geospatial data. In this way, we prefer to use the term "3D geovisualisation". Based on MacEachren and Kraak's definition, the geovisualisation is defined as the field that provides theory, methods, and tools for visual exploration, analysis, synthesis, and presentation of geospatial data (MacEachren and Kraak, 2001). ${ }^{2}$
To establish a standardisation of 3D representation rules, we start the discussion with the 2D cartographic representation rules developed by cartographers over the last fifty years. The next sub-section presents all static visual variables developed on $2 \mathrm{D}$ objects.

\subsection{Visual variables in 2D}

In 1967, Jacques Bertin published a book on the graphic's semiology. This book entitled "Sémiologie graphique" constitutes a major reference in semiotics. In his book, Bertin defines 7 retinal variables used for punctual (points), linear (lines) and zonal (zones) primitives (Bertin, 1967). It is worth noting that the computational displays emergence leads to introduce new primitives: surfaces (comparable to areas except they are located in a 3D scene) and volumes (characterized by a depth contrary to area or surface) (Carpendale, 2003). The visual variables defined by Bertin are:
1. Position (in space);
2. $\quad$ Size (of the symbol);
3. Value: the black and white ratio within an unit surface;
4. Grain (or texture): the number of distinct symbols within an unit surface;
5. Colour (from the visible spectrum);
6. Orientation (between the horizontal and the vertical and characterized by a sense);
7. Shape (of the symbol).

These visual variables have been applied on paper by using 2D symbols (points, lines and polygons). Each retinal variable was studied according to four interpretation tasks (Bertin, 1967):

1. Selectivity: the capacity to extract categories; the question is: does the retinal variable variation enable to identify categories?

2. Associativity: the capacity to regroup similarities; the question is: does the retinal variable variation enable to group similarities?

3. Order perception: the capacity to compare several orders; the question is: does the retinal variable variation enable to identify a change in order?

4. Quantitative perception: the capacity to quantify a difference; the question is: does the retinal variation enable to quantity a difference?

Bertin's graphic semiology contribution to $2 \mathrm{D}$ cartography is unquestionable especially in the decisional cartography by the increasing use of thematic maps. The land-use and land-cover maps incorporate also these visual variables in order to represent categories, sub-categories or to put in order groups (Steinberg, 2000). Bertin's visual variables list is not exhaustive and new authors proposed additional static visual variables over time. In 1974, Morrison introduced the arrangement and the saturation (Halik, 2012). To represent uncertainty, MacEachren proposed in 1995 three retinal variables: the crispness, the transparency and the resolution (MacEachren, 2005). Finally, Slocum et al. introduced in 2010 the spacing and the perspective height. Table 1 summarizes the static visual variables developed over the last fifty years (Halik, 2012). Each variable is characterized by the cartographers who referred to (Halik, 2012). 


\begin{tabular}{|l|l|}
\hline $\begin{array}{l}\text { STATIC VISUAL } \\
\text { VARIABLES }\end{array}$ & AUTHOR (DATE) \\
\hline Size & $\begin{array}{l}\text { Bertin (1967), Morisson (1974), MacEachren (1995), Kraak \& Ormeling (2003), } \\
\text { Krygier \& Wood (2005), Dent et al. (2009), Slocum et al. (2010), Tyner (2010) }\end{array}$ \\
\hline Shape & $\begin{array}{l}\text { Bertin (1967), Morisson (1974), MacEachren (1995), Kraak \& Ormeling (2003), } \\
\text { Krygier \& Wood (2005), Dent et al. (2009), Slocum et al. (2010), Tyner (2010) }\end{array}$ \\
\hline Value & $\begin{array}{l}\text { Bertin (1967), Morisson (1974), MacEachren (1995), Kraak \& Ormeling (2003), } \\
\text { Krygier \& Wood (2005), Dent et al. (2009), Slocum et al. (2010), Tyner (2010) }\end{array}$ \\
\hline Colour (hue + saturation) & Bertin (1967) \\
\hline Orientation & $\begin{array}{l}\text { Bertin (1967), Morisson (1974), MacEachren (1995), Kraak \& Ormeling (2003), } \\
\text { Krygier \& Wood (2005), Dent et al. (2009), Slocum et al. (2010), Tyner (2010) }\end{array}$ \\
\hline Texture & $\begin{array}{l}\text { Bertin (1967), Morisson (1974), MacEachren (1995), Kraak \& Ormeling (2003), } \\
\text { Krygier \& Wood (2005), Dent et al. (2009), Tyner (2010) }\end{array}$ \\
\hline Position & $\begin{array}{l}\text { Bertin (1967), MacEachren (1995), Kraak \& Ormeling (2003), } \\
\text { Krygier \& Wood (2005), Dent et al. (2009), Slocum et al. (2010), Tyner (2010) }\end{array}$ \\
\hline Hue & $\begin{array}{l}\text { Morrison (1974), MacEachren (1995), , Kraak \& Ormeling (2003), } \\
\text { Krygier \& Wood (2005), Dent et al. (2009), Slocum et al. (2010), Tyner (2010) }\end{array}$ \\
\hline Saturation & $\begin{array}{l}\text { Morrison (1974), MacEachren (1995), Krygier \& Wood (2005), Dent et al. } \\
\text { (2009), Slocum et al. (2010), Tyner (2010) }\end{array}$ \\
\hline Arrangement & $\begin{array}{l}\text { Morrison (1974), MacEachren (1995), Dent et al. (2009), Slocum et al. (2010), } \\
\text { Tyner (2010) }\end{array}$ \\
\hline Crispness & MacEachren (1995) \\
\hline Transparency & MacEachren (1995) \\
\hline Resolution & MacEachren (1995) \\
\hline Spacing & Slocum (2010) \\
\hline Perspective height & Slocum (2010) \\
\hline Table & Vis 2 (2019) Variables distinguished OVer the last fifty \\
\hline
\end{tabular}

Table 1. Static visual variables distinguished over the last fifty years inspired from Halik (Halik, 2012)

\subsection{Visual variables in 3D}

All these visual variables have been developed on $2 \mathrm{D}$ objects. With the emergence of $3 \mathrm{D}$ environments, new mechanisms (lighting, shading, atmosphere effects, depth of field ...) come out and interfere with the retinal variables visualisation. For instance, the depth of field can influence the perception of size and density while an artificial light source can change the colours perception. Consequently, all the visual variables defined in 2D are not directly transposable in 3D scenes. This is why a standardisation of representation rules must be developed for $3 \mathrm{D}$ geovisualisation. The next paragraphs present studies in the field of 3D semiotics.

In 2005, Foss et al. (2005) considered hue as a visual variable in 3D thematic maps. Based on a virtual model produced with prisms, they analysed the impact of different shadings on 3D objects. They concluded that the light source location and colour selection influence the 3D representation. Indeed, changing the light source location leads to modify the scene illumination. It impacts the colour perception with a variation of saturation which is specific for each colour depending on the natural brightness of the colour.

In 2012, Halik (2012) summarized the current static retinal variables used in 2D cartography. He started a discussion process in the field of augmented reality displayed on smartphones and analysed the efficiency of visual variables according to four interpretation tasks (selectivity, associativity, quantitative and order aspects).

In 2012, Wang et al. (2012) analysed Bertin's visual variables through 3D legal units on the criterion of selectivity for a potential 3D cadastre system. The selectivity is investigated via requirements for 3D cadastre visualisation. From 2012 and 2014, Pouliot et al. analysed the efficiency of different visual variables (hue, saturation, value, texture, position) as well as techniques like transparency, moving elements and labels on selectivity cadastral tasks (Pouliot et al., 2013; Pouliot et al., 2014a; Pouliot et al., 2014b). Based on notaries' interviews, their results highlighted that hue is one of the most encouraging variable for selectivity tasks. It also appeared that transparency can favour the annotations reading (official measures). However, this variable introduces some confusions as soon as too many 3D lots are visible. They also demonstrated that transparency influences the notaries' decision with respect to the distinction of private and common parts or to establish the ownership property. Nevertheless, they could not prove that a certain transparency level was better adapted for the two previous tasks realisation.

In 2015, Rautenbach et al. (2015) assessed visual variables (position, size, shape, value, colour, orientation, texture and motion) with regard to the selectivity in the domain of informal settlements for urban planning. They concluded that in order to increase the selectivity, the camera position, orientation and motion have to be taken into account. However, the camera motion can influence some visual variables like value. They also showed that hue and texture seem to be the most adapted variables for selectivity tasks.

\subsection{Highlighting techniques and multi-perspective views}

As visual variables are analysed in $3 \mathrm{D}$, new techniques appear in order to visualise users' selections or database queries in 3D environments; highlighting techniques and multi-perspective views are consequently useful for the 3D geovisualisation. The highlighting techniques can be classified according to the types of rendering (Trapp et al., 2010):

1. Style-variance techniques; this technique consists in highlighting an object by changing its appearance (focus-based style variance technique) or the context appearance (context-based style variance technique).

2. Outlining techniques; this technique consists in highlighting the object outline or the silhouette.

3. Glyph-based techniques; this technique consists in highlighting the object by adding icons or glyphs to this object.

The context-based style variance and the outline techniques appear to be the most relevant techniques since they enable (to some extent) to highlight hidden objects in the scene (Trapp et al., 2010).

To enhance the 3D environment view in terms of relayed information by reducing the number of hidden objects, new techniques are currently developing like multi-perspective views. These techniques are based on panorama maps and exist in two views: the bird's eye view and the pedestrian's view. The bird's eye view is characterized by a top view in the foreground and a ground view in the background unlike the pedestrian's view which combines a top view in the background and a ground view in the foreground. Both views present a smooth transition zone between the foreground and the background (Lorenz et al., 2008). Independently of bird's eye view and top view, the perspective view selection can also reduce the number of dead values which are pixels that cannot transfer information about their content. Unlike central perspectives (characterized by a linear perspective and multiple scales in one view), parallel perspectives present only one scale which enables to compare objects sizes and orientation but modifies the naïve perceptions (Jobst and Döllner, 2008). 


\section{VIEWPOINT MANAGEMENT}

\subsection{Needs and resolution}

Whilst visual variables enable to relay information (thematic, topological ...) regarding to objects (either 2D or 3D), they are only useful if the objects are visible. In this way, camera management is essential. A non-optimal camera management can induce a partial view of the scene independently of the representation of objects. Let's imagine that we want to observe some apartments in a building (e.g. according to the number of rooms). Depending on the viewpoint, the number of seen apartments can significantly vary which implies that some views are better suited. Indeed, providing a view where none apartment is seen has no interest in terms of communication. Pegg (2009) and Rautenbach et al. (2015) conclude that the camera location is an essential aspect in 3D. Certainly, transparency, highlighting techniques and multi-perspective views enable to increase the visibility of hidden objects and are consequently useful regarding the occlusion management (Lorenz et al., 2008; Trapp et al., 2010; Pouliot et al., 2014a; Pouliot et al., 2014b). Nevertheless, the final viewpoint of the 3D scene will constitute the fundamental parameter that will act on the visibility of objects. The interrelations between occlusion management techniques and the viewpoint are going to be studied (further) in a next research step.

In a 3D environment, the number of degrees of freedom relating to the viewpoint increases compared to $2 \mathrm{D}$. Indeed, the camera can be located in space according to three coordinates: $\mathrm{x}, \mathrm{y}$ and z. Consequently, the viewing direction is not only oriented in a top down direction. In this way, 3D semiotics structure incorporates the camera management defined as a variable of vision (Jobst et al., 2008). The camera management includes the camera location, the viewing direction and the perspective. All of these aspects are essential in 3D scenes and by extension in 3D maps since maps are means of communication which relay information.

The viewpoint management is therefore the first step towards a normalised 3D geovisualisation. We support the idea that the viewpoint is a fundamental parameter making the use of visual variables relevant to relay information. That is why we consider the viewpoint management before the analysis of 2D static visual variables. To address the viewpoint efficiency, we developed a demonstrator staging a Rubik's cube that will serve for end-users testing at a later stage. To determine the optimal viewpoint, we propose a method based on the pixels of the end-user computational display. At this stage, the method aims only at providing an operational solution for the demonstrator; depending on the type of 3D data structure, other solutions could be applied. A comprehensive study of the method performances is out of the scope of this paper. The following sub-sections present a case study dealing with the viewpoint and the obtained results.

\subsection{Demonstrator}

\subsubsection{Development}

We designed a Rubik's cube constituted of 27 individual cubes that correspond to the objects of the $3 \mathrm{D}$ environment. Each cube is characterized by one of the following colour: red, green, blue, white, orange or yellow. It is worth noting that edges of cubes are represented in black in order to visualize their spatial extend. The objective of this demonstrator is to propose an observer viewpoint so that he can observe a maximum of cubes of selected colour. The user can then move in real time in the scene with the keyboard and the mouse and can observe the cube from different viewing angles. It is worth mentioning that we only consider the observation queries at this stage. We do not process tasks like target discovery, access or relation.

We propose a method based on a pixels analysis of the final computational display. Processing screen pixels enables to work on the final rendering that already incorporates the management of hidden faces by the use of a processing algorithm for hidden faces (e.g. Z-Buffer). In order to treat screen pixels, we directly work with the graphic card of the computer. The prototype has been designed thanks to two libraries: OpenGL (Open Graphics Library) and $S D L$ (Simple Directmedia Layer). The first library runs with the graphic card and is used to create a representation of a Rubik's cube in three dimensions (see figure 2). The second library is used to create a window in which the Rubik's cube appears and to manage the keyboard and the mouse in order to move in the 3D scene. These two libraries present some advantages as being multiplatform (running on Windows, Mac and Linux) and opensource (OpenGL, 1997; SDL, 1998). Finally, the programming language used to create the prototype is $\mathrm{C}++$.

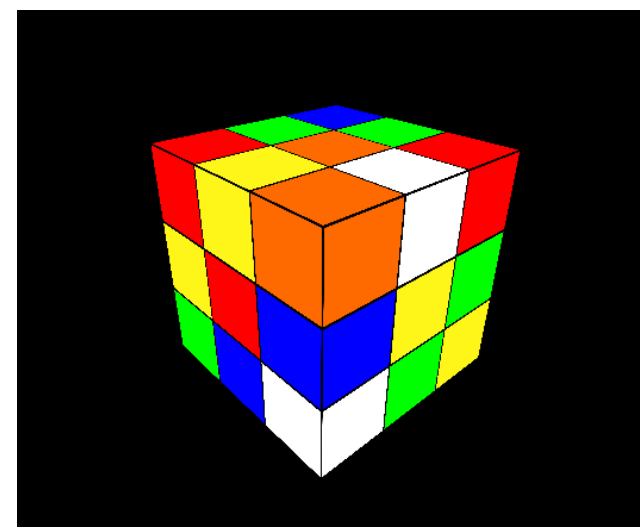

Figure 2. Rubik's cube view

Black edges represent the spatial extension of cubes

The Rubik's cube is located at the origin of the three axis (X, Y and $\mathrm{Z}$ ) and is positioned symmetrically according to these axis (see figure 3 ). The camera is positioned on a half-sphere centred at the origin of the axis and moves with 15 degrees increments. This number is fixed arbitrary due to computation requirements but can easily change upon the user's needs. The more the steps, higher the calculation time. Moreover, a small location difference between two camera positions is not always relevant with respect to the maximisation of objects representation. A half-sphere is considered in order to only obtain views taken from the top. The radius of the sphere has been determined to see the whole Rubik's cube whether the camera position. 


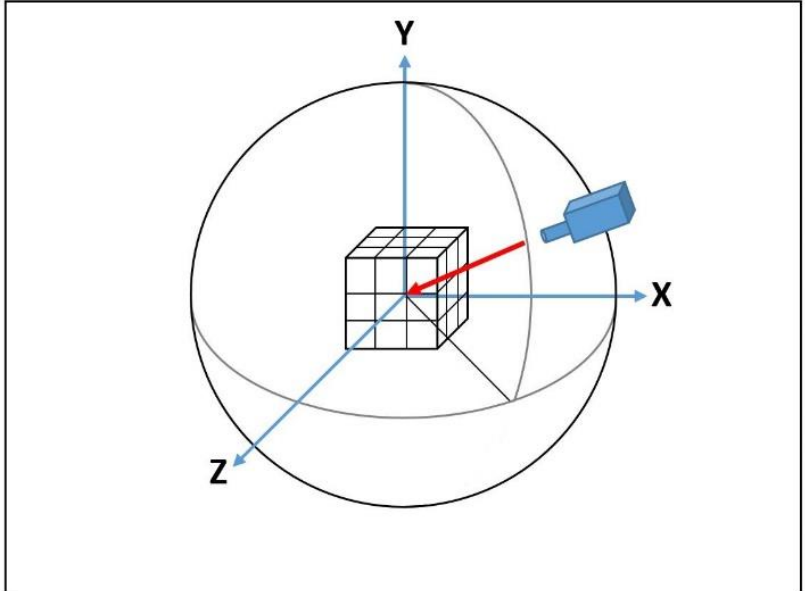

Figure 3. Camera management

For each camera position, an image of the Rubik's cube is generated on the screen in order to count the number of distinct objects (which correspond to cubes of a certain colour) seen from this camera position. The visibility of objects is processed by a Z-buffer algorithm provided by OpenGL; other techniques or algorithms could have been used: e.g. ray-tracing. Nevertheless, the core of the method remains the pixels processing after the use of an algorithm or technique to process the hidden faces.

The image resolution corresponds to the resolution of the screen and the size of the image is smaller than the screen size (only 50 pixels in width and 50 pixels in height) in order to improve the performance by reducing the computation time. A based on screen pixels colour algorithm has been developed to determine the number of objects. The optimal camera position which maximises the number of objects is obtained after an iterative process in which the camera moves according to a half sphere. After a horizontal and vertical angle (respectively $\Theta$ and $\phi)$ from the initial positon, the coordinates $\mathrm{X}, \mathrm{Y}$ and $\mathrm{Z}$ are obtained by the following formula (if the horizontal angle is non-null):

$$
\begin{aligned}
& \mathrm{X}=\mathrm{r} * \cos (\theta) * \cos (\phi) ; \\
& Y=\mathrm{r} * \sin (\phi) ; \\
& Z=\mathrm{r} * \cos (\phi) * \sin (\theta) ;
\end{aligned}
$$

where $r=$ radius;

$$
\Theta=\text { horizontal angle; }
$$$$
\phi=\text { vertical angle; }
$$

$\mathrm{X}, \mathrm{Y}, \mathrm{Z}=$ camera coordinates.

\subsubsection{Objects detection Algorithm}

Based on a region growing process (Tremeau and Borel, 1996), we have developed a colour algorithm to determine the number cubes of a certain colour. For each generated image, every pixel of the end-user computational display is read line by line starting from the bottom left pixel. It is worth specifying that the algorithm is applied on a Rubik's cube where the edges are no longer represented. In this way, we define an object as a cube and not a face of cube. The environment surrounded each pixel is analysed to determine if this pixel starts a new object (a cube of a specific colour) or belongs to an already detected object. The pixel neighbourhood is defined by the eight pixels around the analysed pixel. To detect a new object, we analyse the three pixels located below the current pixel but also the right pixel (see figure 4). If all these pixels have a different colour, we detect a new object (cube) and we increment the objects counter for the view. Otherwise, if the right pixel has the same colour than the current pixel, we search a link between the current pixel or the same colour adjacent pixels (located on the same line) and a neighbour pixel located on the previous line. Indeed, all neighbour pixels on the previous line have been already read; they could eventually have already defined the object linked to the current pixel or the same colour adjacent pixels. If no link appears, a new object is detected.

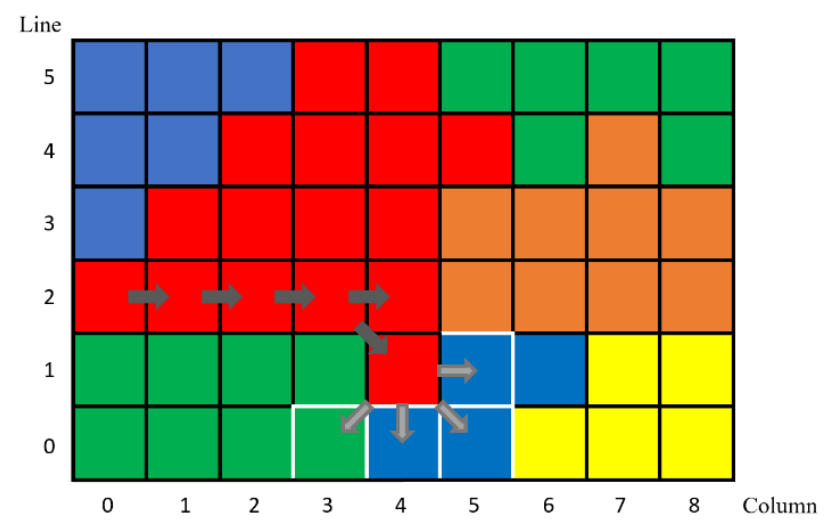

Figure 4. Objects detection algorithm on the pixels of the enduser computational display

Figure 4 shows an example of the algorithm with red cubes. The pixel located at line 1 and at column 4 is the first read red pixel since the reading starts from the bottom left pixel. Therefore, we analyse the pixels located below the current pixel but also the right pixel. As all these neighbourhood pixel are not red, we detect a new object. Let's now move on line 2 and column 0 . As the right pixel (line 2 and column 1) is red, an iterative process starts with all adjacent red pixels located at this line. For each pixel (from column 0 to 4), we analyse the adjacent pixels of the previous line to detect an eventually red pixel located on line 1 . Indeed, all pixels on this line have been already read and therefore they eventually could already defined an object linked to the analysed pixels. In this example, the connection with an already defined object is performed at the pixel located at line 2 and column 3. Indeed, this pixel presents a link with one of its neighbourhood pixels; the pixel located at the previous line and at the next column. All red pixels of the line 2 do not define a new object but they belong to an already detected object (defined in line 1 and column 4).

\subsection{Results}

The objects detection algorithm has been applied for each colour of the Rubik's cube. Figure 5 represents the optimal view for the orange colour; four objects are seen from this view. The position camera has been computed in a short time period. As already mentioned, the computation time is a function of the step between two camera locations ( 15 degrees in our case) and depends also on the resolution of the display. In order to reduce the computation time, we have used a lower resolution (50 pixels in width and height) than the window resolution (800 pixels in width and 600 in height). Such a resolution enables to observe all parts of the Rubik's cube and to drastically reduce the computation time. Therefore, the whole numerical display resolution is not necessary and a reduction of the number of pixels can be performed according the visibility defined by the user. 


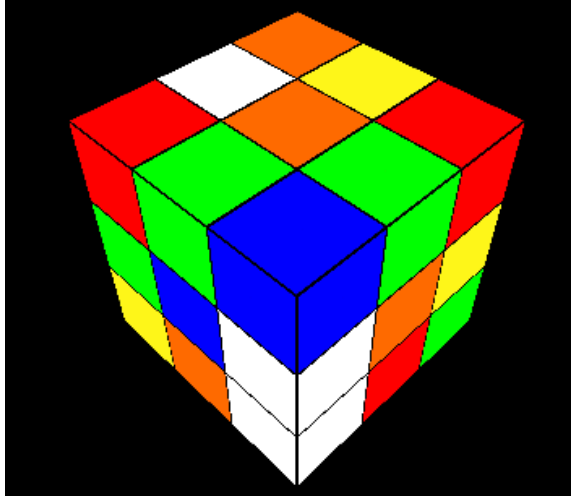

Figure 5. Orange cubes view

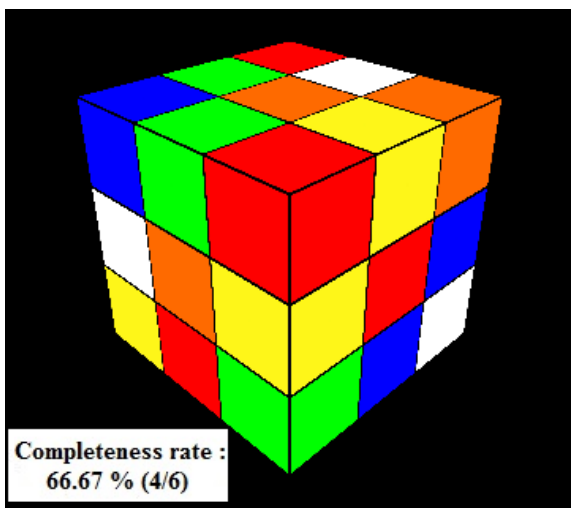

Figure 6. Red cubes view

Unlike 2D, 3D representations deal with hidden faces due to occlusion. That is why a new piece of information should appear on 3D representations: the completeness rate of viewed objects. This rate represents the percentage of objects seen from a certain viewpoint. It is completed by the ratio of seen objects expressed in numbers. Figure 6 represents the optimal view for the red colour; four objects are seen from this view. The completeness rate is equal to $66.67 \%$ since only 4 objects, i.e. small red cubes, on a total of 6 are represented. This rate is an essential element for the understanding of information conveyed by any 3D representations; it allows users to be aware of the limitation of the proposed view. Consequently, the completeness rate constitutes a new element of the cartographic design for $3 \mathrm{D}$ maps.

\section{DISCUSSION}

Based on the demonstrator, we show the impact of the viewpoint on the visualisation of 3D objects. Independently of used visual variables (only hue in our case), an optimal viewpoint enables to increase the relayed information regarding the visualisation of coloured cubes (see figure 5 and 6 ).

Nevertheless, it is worth noting that all parts of the Rubik's cube have been coloured entirely which makes impossible to observe the central cube. Moreover, the cube below the central cube cannot be seen too as we consider only views from the top. This limit could be solved by using other visual variables for the nonrequested objects: e.g. transparency. This retinal variable will certainly influence the proposed viewpoint for red cubes. However, the supported idea remains the same. At the end of the process of retinal variable(s) selection, the viewpoint is the fundamental parameter that will act on the visualisation of $3 \mathrm{D}$ objects. In this paper, only maximisation (of 3D objects) criterion has been taken into account. Other criteria could also be used depending on the application fields and consequently viewing tasks. For instance, the camera viewing angle could be combined or preferred to the objects maximisation in the context of urban visualisation for pedestrians.

Regarding the objects detection algorithm, the camera location based on screen pixels presents a substantial advantage. The developed method is actually independent from the type of data (vector, raster or point cloud) since we work on the pixels of the final computational display. The optimal viewpoint in terms of relayed information can be found for each of these formats.

The method presents also some disadvantages. The computation time is certainly one of the principal limit for a treatment in real time. It is due to the computation of significant images. However, it is worth noting that the process time can be decreased by the use of more efficient functions in OpenGL. Moreover, we have developed a based on screen pixels colour algorithm meaning that we only consider the colorimetric information of each pixel to identify and count objects. To improve the performance, we should instead work on the object identifier to which a pixel belongs. Then all we have to do is to count the number of distinct objects on the screen. It is worth mentioning that a minimum number of pixels should be determined for any represented objects in order to insure the object visibility in the 3D scene. In the case of two or more views depict the same number of objects, we should keep the one that maximises the number of seen pixels for the requested objects. Another parameter should also be taken into account: the distribution of pixels according the objects. Indeed, views characterized by the dominance of one object (like a building in a city) should be avoided in favour of views which balances the number of viewed pixels for each object.

\section{CONCLUSION AND FUTURE WORK}

The recent advances in computer sciences (in terms of storage and processing of large amount of data) and the growing number of acquisition techniques (LiDAR, photogrammetry and remote sensing) led to impressive improvements in the 3D visualisation. Numerous application fields use 3D models which implies to develop a standardisation of 3D representation rules. The need of standardisation relates to both 3D cartography and also 3D applications located in the virtuality continuum that include geospatial data. This is why we use the term $3 \mathrm{D}$ geovisualisation.

To establish a normalised 3D geovisualisation, we start the discussion with the 2D cartographic representation rules developed by cartographers over the last fifty years. 3D environments include new mechanisms (e.g. depth of field, lighting, shading ...) that interfere with the visualisation of retinal variables developed on 2D objects. Therefore, all the visual variables defined in $2 \mathrm{D}$ are not directly transposable in $3 \mathrm{D}$ scenes. As a consequence, some studies must be conducted in order to establish a normalised $3 \mathrm{D}$ geovisualisation.

As a first step towards a normalised 3D geovisualisation, we tackle the viewpoint management. Unlike 2D environments, the camera management becomes an issue in all 3D representations including an allocentric environment since the camera is not only fixed in a top down direction. A non-optimal camera management can imply a partial view of the scene independently of the representation of objects. Consequently, we support the idea that the viewpoint is a fundamental parameter making the use of visual variables relevant to relay information.

To address the viewpoint efficiency, we developed a demonstrator staging a Rubik's cube (whose components are 
coloured cubes) that will serve for end-users testing at a later stage. The objective of this demonstrator is to propose an observer viewpoint so that he can observe a maximum of cubes of a selected colour. To achieve this objective, we proposed a method based on the pixels of the end-user computational display. We move the camera according to an upper half-sphere centred at the centre of the Rubik's cube. For each camera position, an image of the Rubik's cube is generated. The image resolution corresponds to the resolution of the screen. To calculate the number of objects on each image, we have developed a based on pixels (of the screen) colour algorithm. At the end of the process, the algorithm gives the three-dimensional coordinates of the camera that maximises the relayed information concerning a specific colour.

The demonstrator shows that an optimal viewpoint enables to increase the relayed information with regard to the visualisation of coloured cubes. As constructed, the Rubik's cube presents an important limitation: the impossibility to observe the central cube and the one just below. This limit could be solved by the use of other visual variables for the non-requested objects (e.g. transparency). Whilst it will certainly impact on the optimal camera location, the viewpoint will remain the fundamental parameter at the end of the process of retinal variable(s) selection that will act on the visualisation of 3D objects. Finally, the maximisation (of 3D objects) is only one among several criteria to manage the viewpoint. Depending on the application field, other criteria could be used: e.g. camera viewing angle, pixels distribution according to the objects, etc.

The camera management based on the end-user computational display pixels presents a substantial advantage: the independence from the type of data (vector, raster or point cloud). The method presents also some disadvantages like being colorimetric information-oriented which leads to increase the computational time by the use of a specific objects detection algorithm. To enhance the performance, we should work with the object identifier to which a pixel belongs.

Through the viewpoint management, this paper constitutes a first step towards a normalised 3D geovisualisation. Future research will follow to continue the analysis of visual variables and parameters used in 3D applications that include geospatial data and therefore our work of standardisation.

\section{REFERENCES}

Bandrova, T., 2005. Innovative Technology for the Creation of 3D Maps. Data Science Journal, Vol. 4, pp. 53-58.

Bertin, J., 1967. Sémiologie graphique: les diagrammes, les réseaux et les cartes, Paris, Mouton/Gauthier-Villars.

Behzadan, A. H., Suyang D., \& Vineet R. K., 2015. Augmented Reality Visualization: A Review of Civil Infrastructure System Applications. Advanced Engineering Informatics, 29(2), pp. 252-267.

Carpendale, M. S. T., 2003. Considering Visual Variables as a Basis for Information Visualisation. Dept. of Computer Science, University of Calgary, Canada, Tech. Rep. 2001-693-16. http://dspace.ucalgary.ca/bitstream/1880/45758/2/2001-69316.pdf

Egenhofer, M. J., and Mark, D. M., 1995. Naive Geography. In A. Frank \& W. Kuhn (Eds.). Spatial information theory: A theoretical basis for GIS, COSIT' 95, Vol. 988 Lecture Notes in Computer Science, Berlin: Springer, pp 1-15.
Ellul, C. \& Altenbuchner, J., 2014. Investigating Approaches to Improving Rendering Performance of 3D City Models on Mobile Devices. Geo-Spatial Information Science, 17(2), pp. 73-84.

Fosse, J.M., Veiga, L.A.K. \& C.R. Sluter., 2005. Color Hue as a Visual Variable in 3D Interactive Map. In IEEE International Conference on Communications, Vol. 55, Seoul, Korea.

Häberling, C., \& Baer, H.R., 2006. Aspects of 3D Map Integration in Interactive School Atlases. In 5th ICA Mountain Cartography Workshop, Bohinj, Slovenia.

Häberling, C., Bär, H. \& Hurni, L., 2008. Proposed Cartographic Design Principles for 3D Maps: A Contribution to an Extended Cartographic Theory. Cartographica: The International Journal for Geographic Information and Geovisualization, 43(3), pp.175-188.

Halik, L., 2012. The Analysis of Visual Variables for Use in the Cartographic Design of Point Symbols for Mobile Augmented Reality Applications. In Geodesy and Cartography 61(1), pp. 19-30.

Jobst, M., \& Döllner, J., 2008. Better Perception of 3D-Spatial Relations by Viewport Variations. M. Sebillo, G. Vitiello, G. Schaefer (Eds.), Visual Information Systems. Web-Based Visual Information Search and Management, Lecture Notes in Computer Science, Springer-Verlag, pp. 7-18.

Jobst, M., \& Germanchis, T., 2007. The Employment of 3D in cartography-An Overview. W. Cartwright, M. P. Peterson, G. Gartner (Eds.), Multimedia Cartography, Springer-Verlag, pp.217-228.

Jobst, M., Kyprianidis, J.E., \& Döllner, J., 2008. Mechanisms on Graphical Core Variables in the Design of Cartographic 3D City Presentations. A. Moore, I. Drecki (Eds.), Geospatial Vision, Lecture Notes in Geoinformation and Cartography, SpringerVerlag, pp. 45-59.

Lorenz, H., Trapp, M., Döllner, J. \& Jobst, M., 2008. Interactive Multi-Perspective Views of Virtual 3D Landscape and City Models. L. Bernard, A. Friis-Christensen, H. Pundt (Eds.), The European Information Society, Lecture Notes in Geoinformation and Cartography, Springer-Verlag, pp. 301-321.

MacEachren, A. M., and Kraak, M.-J., 2001. Research Challenges in Geovisualization. Cartography and Geographic Information Science, 28(1), pp. 3-12.

MacEachren, A.M., Robinson, A., Hopper, S., Gardner, S., Murray, R., Gahegan, M. \& Hetzler, E., 2005. Visualizing Geospatial Information Uncertainty: What We Know and What We Need to Know. Cartography and Geographic Information Science, 32(3), pp. 139-160.

Métral, C., Ghoula, N., Silva, V. \& Falquet, G., 2014. A Repository of Information Visualization Techniques to Support the Design of 3D Virtual City Models. U. Isikdag (Ed.), Innovations in 3D Geo-Information Sciences, Lecture Notes in Geoinformation and Cartography, Springer, pp. 175-194.

Milgram, P. \& Kishino, F., 1994. A Taxonomy of Mixed Reality Visual Displays. IEICE TRANSACTIONS on Information and Systems, 77(12), pp. 1321-1329.

Milgram, P., Takemura, H., Utsumi, A. \& Kishino, F., 1995. Augmented Reality: A Class of Displays on the Reality- 
Virtuality Continuum. In Photonics for Industrial Applications, International Society for Optics and Photonics, pp. 282-292.

OpenGL, 1997. https://www.opengl.org/, last visit the 2016/05/19.

Pegg, D., 2009. Design Issues with 3D Maps and the Need for 3D Cartographic Design Principles. Academic Paper. http://lazarus.elte.hu/cet/academic/pegg.pdf (May 2016).

Petrovič, D. \& Mašera, P., 2005. Analysis of User's Response on 3D Cartographic Presentations. In the Proceedings of the 22nd ICA International Cartographic Conference, A Coruña, Spain.

Pouliot, J., Wang, C., Fuchs, V., Hubert, F. \& Bédard, M., 2013. Experiments with Notaries about the Semiology of 3D Cadastral Models. In: The International Archives of the Photogrammetry, Remote Sensing and Spatial Information Sciences, Istanbul, Turkey, Vol. XL-2/W2, p53-57.

Pouliot, J., Wang, C. \& Hubert, F., 2014a. Transparency Performance in the 3D Visualization of Bounding Legal and Physical Objects: Preliminary Results of a Survey. In the Proceedings of the 4th International Workshop on 3D Cadastres, Dubai, pp. 173-182.

Pouliot, J., Wang, C., Hubert, F. \& Fuchs, V., 2014b. Empirical Assessment of the Suitability of Visual Variables to Achieve Notarial Tasks Established from 3D Condominium Models. U. Isikdag (Ed.), Innovations in $3 D$ Geo-Information Sciences, Lecture Notes in Geoinformation and Cartography, Springer International Publishing Switzerland, pp. 195-210.

Rautenbach, V., Coetzee, S., Schiewe, J. \& Çöltekin, A., 2015. An Assessment of Visual Variables for the Cartographic Design of 3D Informal Settlement Models. In the Proceedings of the ICC 2015, Rio de Janeiro, Brazil.

SDL, 1998. https://www.libsdl.org/, last visit the 2016/05/19.

Steinberg, J., 2000. L'apport de La Sémiologie Graphique de Jacques Bertin a La Cartographie Pour L'aménagement et L'urbanisme. Cybergeo: European Journal of Geography.

Terribilini, A., 2001. Entwicklung von Arbeitsabläufen zur automatischen Erstellung von interaktiven, vektorbasierten topographischen 3D-Karten. Institute of Cartography, ETH Zurich.

Trapp, M., Beesk, C., Pasewaldt, S. \& Döllner, J., 2010. Interactive Rendering Techniques for Highlighting in 3D Geovirtual Environments. In the Proceedings of the $5^{\text {th }} 3 D$ geoInfo Conference, XXXVIII, 12669-12669.

Tremeau, A., Borel, N., 1996. A region growing and merging algorithm to color segmentation. Pattern Recognition, 30(7), pp.1191-1203.

Wang, C., Pouliot, J. \& Hubert, F., 2012. Visualization Principles in 3D Cadastre: A First Assessment of Visual Variables. In the Proceedings of the 3 rd International Workshop on $3 D$ Cadastres, Shenzhen, pp. 309-324. 